\title{
Baade-Wesselink Analysis of Type II Cepheids
}

\author{
J. Vinkó and Z. Balog \\ Department of Optics, JATE University, Szeged, H-6720 Hungary, \\ e-mail:vinko@physx.u-szeged.hu
}

\begin{abstract}
New physical parameters of selected Cepheid variables classified as CW or CEP (Type II) in GCVS are presented. The analysis is based on published light and radial velocity curves. Two of the stars turned out to be classical Cepheids.
\end{abstract}

The short period metal-rich variables form an interesting group among Type II Cepheids (also called metal-rich BL Her-type stars). Their kinematic properties indicate that they differ from the normal disk population, because of e.g., their high galactic latitude. On the other hand, their chemical abundances are much closer to Pop. I composition. Moreover, from theoretical point of view, it is not clear yet how these stars can evolve into the instability strip and become pulsating stars.

It is known that Type I and II Cepheids are difficult to distinguish (Harris, 1985), because absolute physical parameters are not easy to obtain due to mainly the faintness of Type II Cepheids. Recently some new radial velocity curves have become available (e.g., from Gorynya et al., 1992) which enabled us to compute Baade-Wesselink radii for five selected Type II Cepheids which are relatively well observed and have nearly solar metallicity, $[\mathrm{Fe} / \mathrm{H}]$ close to 0 . The resultant radius values were used to calculate other physical parameters via theoretical period-mass-radius and mass-luminosity relations for both populations.

All of these results are collected in Table 1, while Fig. 1 shows the position of the variables in the $H R$-diagram (filled symbols). Fig. 1 also contains reference data for both Pop. I and Pop. II objects: some known Cepheids in galactic open clusters and several short period BL Her-type stars in globular clusters (open squares and circles, respectively).

The conclusions are the following. TX Del and V351 Cep are probably misclassified as CW (Type II Cepheid), because they are too large and hence too bright to be Type II Cepheids. These results strongly suggest their Pop. I nature, because they are in the region of Classical Cepheids. Moreover, V351 Cep is probably an s-Cepheid which supports its Pop. I classification. TX Del is actually a binary Cepheid with a low mass function (Harris \& Welch, 1989), which gives $M_{2}(\mathrm{~min})=1.1 M_{\odot}$ for the companion. The Roche-lobe filling factor in this case becomes $\mathrm{f}=0.44$. The Pop.I nature of TX Del is also supported by its infrared light curves (Laney, 1995). Note that the galactic z-distance of TX Del is much larger than most of the classical Cepheids.

IX Cas is also close to the regime of classical Cepheids; however, its physical parameters indicate later evolutionary state (e.g., its mass is too low). BL Her, the prototype of this class of variables, is clearly among Pop. II objects. AU 


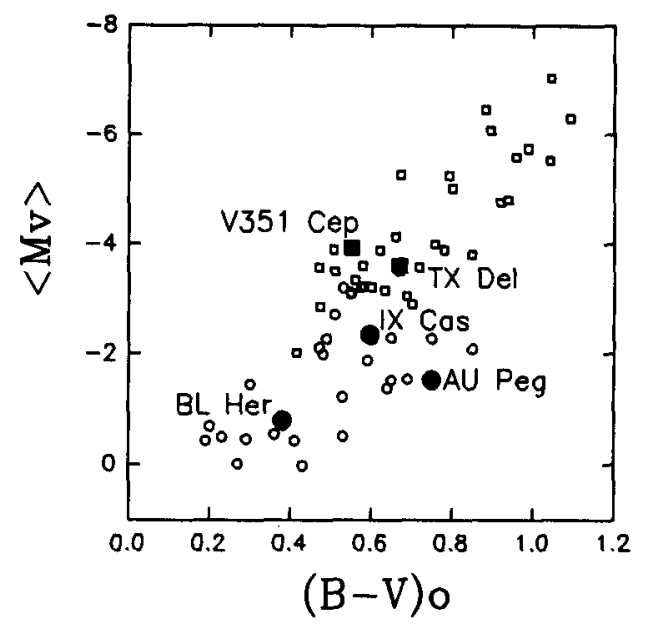

Figure 1. The position of the variable in the HR diagram. Open symbols represent reference Cepheids of both types (see text).

Peg, another binary Cepheid, is peculiar in many respects (e.g., Vinkó, Szabados \& Szatmáry, 1993). Referring to Table 1, it is more massive than most of the Pop. II Cepheids (which usually have $M<1 M_{\odot}$ ). On the other hand, AU Peg does not resemble any classical Cepheids, therefore its classification is uncertain.

Table 1. Inferred physical parameters of the program stars.

\begin{tabular}{cccccc} 
Star & $R / R_{\odot}$ & $M / M_{\odot}$ & $M_{v}$ & $d(k p c)$ & $z(k p c)$ \\
\hline BL Her & 9.4 & 0.8 & -0.78 & 1.43 & 0.47 \\
AU Peg & 20.0 & 2.3 & -1.60 & 1.28 & 0.48 \\
IX Cas & 24.0 & 0.5 & -2.29 & 4.81 & 0.99 \\
TX Del & 48.0 & 5.9 & -3.54 & 2.98 & 1.22 \\
V351 Cep & 49.6 & 6.3 & -3.89 & 2.58 & 0.03
\end{tabular}

This work has been supported by the Hungarian OTKA Grant \#F7318.

\section{References}

Gorynya, N.A., Irsmambetova, T.R., Rastorgouev, A.S., \& Samus, N.N. 1992, Pisma A.Zh., 18 No.9. 777

Harris, H.C. 1985, AJ, 90, 756

Harris, H.C., Welch, D.L. 1989, AJ, 98, 981

Laney, C.D. 1995, in this proceedings

Vinkó, J., Szabados, L., \& Szatmáry, K. 1993, A\&A, 279, 410 